\title{
Review Article \\ Phenotypic Plasticity and Selection: Nonexclusive Mechanisms of Adaptation
}

\author{
S. Grenier, P. Barre, and I. Litrico \\ INRA, UR004, P3F, RD 150, Site du Chêne, BP 86006, 86600 Lusignan, France \\ Correspondence should be addressed to I. Litrico; isabelle.litrico@lusignan.inra.fr
}

Received 8 December 2015; Revised 5 April 2016; Accepted 3 May 2016

Academic Editor: Ralf Mrowka

Copyright (c) 2016 S. Grenier et al. This is an open access article distributed under the Creative Commons Attribution License, which permits unrestricted use, distribution, and reproduction in any medium, provided the original work is properly cited.

\begin{abstract}
Selection and plasticity are two mechanisms that allow the adaptation of a population to a changing environment. Interaction between these nonexclusive mechanisms must be considered if we are to understand population survival. This review discusses the ways in which plasticity and selection can interact, based on a review of the literature on selection and phenotypic plasticity in the evolution of populations. The link between selection and phenotypic plasticity is analysed at the level of the individual. Plasticity can affect an individual's response to selection and so may modify the end result of genetic diversity evolution at population level. Genetic diversity increases the ability of populations or communities to adapt to new environmental conditions. Adaptive plasticity increases individual fitness. However this effect must be viewed from the perspective of the costs of plasticity, although these are not easy to estimate. It is becoming necessary to engage in new experimental research to demonstrate the combined effects of selection and plasticity for adaptation and their consequences on the evolution of genetic diversity.
\end{abstract}

\section{Introduction}

How do populations adapt to their environment? This is a crucial question in evolutionary biology and greater knowledge in this field is essential if we are to understand and preserve populations in a changing environment. Adaptation is the sum of the morphological, physiological, and behavioural changes of individuals that allows maintenance of individual fitness and so leads to population and species persistence. These changes arise as a result of two mechanisms operating at the level of the individual: selection and phenotypic plasticity. Under the first mechanism, genetic selection, the frequency of favourable alleles increases over several generations while that of unfavourable ones tends to decrease. The evolution of allele frequencies results from different reproductive efficiencies (fitness between genotypes) and so tends to reduce the genetic diversity within a population. The second mechanism, phenotypic plasticity, is defined as the ability of a genotype (individual) to express different phenotypes according to its environment [1]. A number of methods exist to quantify phenotypic plasticity with the use of various indices such as the trait mean, the trait variation coefficient, the trait reaction norm, and the trait extreme values and phenotypic distances [2]. Through phenotypic plasticity, the plastic traits of individuals are modified without modifying the genetic diversity of the population. But phenotypic plasticity can influence the fitness of individuals $[3,4]$ and be the target of selection [5-7]. The intensity of selection for plasticity depends on the balance between a positive effect and a negative one, the cost of plasticity on fitness. This balance can lead to different levels of genetic diversity within an adapted population [8-10] (Figure 1). Genetic diversity is essential in the long term, since it increases the ability of populations or communities to adapt to new environmental conditions [11-13]. However, despite the systematic coexistence of plasticity and selection in natural populations which encounter different environments (spatial and/or temporal changes) [14-16], there is a lack of experimental information on their combined influence on the evolution of the genetic diversity of populations. The purpose of this paper is to review current knowledge on the consequences of selection and phenotypic plasticity on the genetic diversity of populations and to propose procedures to overcome the limitations of experimental data in order to identify these consequences. 


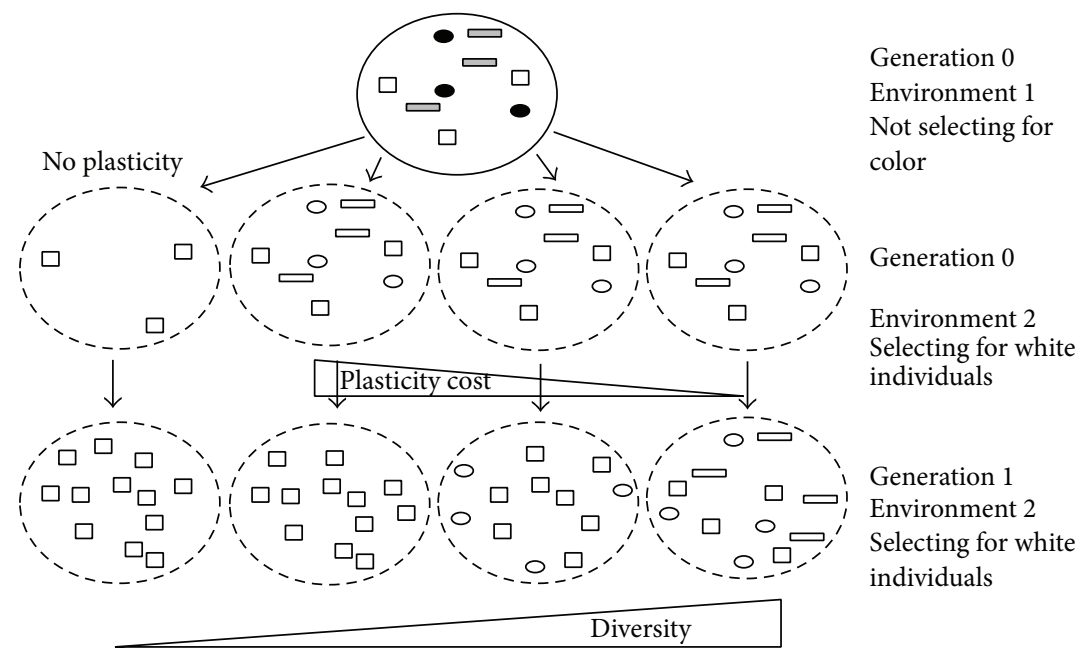

(a)

(b)

(c)

(d)

FIGURE 1: Impact of selection and plasticity on genetic diversity according to the cost of plasticity. Different forms represent genetic diversity and different colours represent different phenotypes and the population size is kept constant. (a) Without plasticity: strong decline of genetic diversity; (b) with equal plasticity among genotypes and a very strong cost of plasticity: strong decline of genetic diversity; (c) with equal plasticity among genotypes and a medium cost of plasticity for the circles and strong cost for the rectangles: low decline of genetic diversity; (d) with equal plasticity among genotypes and no cost of plasticity: maintenance of genetic diversity.

\section{The Consequences of Selection on Genetic Diversity}

Selection occurs on a trait only if it exhibits genetic variability within a population. Selection acts on different character types, physiological processes (e.g., resistance of plants to metals [21]), behaviours (e.g., predator avoidance [22, 23]), or morphological variations (e.g., industrial melanism of the peppered moth [24]). At the phenotypic level, selection changes the mean and/or the variance of trait distributions within a population. Selection can be of three types: directional, stabilising, or disruptive. Given a normal distribution of a trait, directional selection favours individuals with a phenotype either above or below the population mean and so leads to a shift of the mean in one direction or other [25]. Stabilising selection eliminates individuals with the most divergent phenotypes leading to a decrease in trait variance [26]. Disruptive selection favours individuals with extreme phenotypes, leading to a bimodal population distribution [27].

Response to selection results in allele frequency changes of genes involved in the phenotypic variation of traits implicated in individual fitness or at loci in linkage disequilibrium with the previous genes (hitchhiking). In addition to changes in allele frequencies of specific loci, selection can change the allele frequencies globally on the genome by genetic drift, due to a decrease in the effective size of the population. A strong selection can result in a rapid allele fixation (homogenisation of all individuals) and loss of other alleles. This can have a dramatic impact on the genetic diversity of a population (Figure 1). However, selection can also maintain alleles at intermediate frequencies through heterozygote advantage (individuals having two different alleles have greater fitness), or frequency dependence (the allele advantage depends on its frequency relative to other alleles in a given population).
Directional selection tends to decrease the genetic variability of a population in a given environment. But if several populations in different environments are considered (a metapopulation), the decrease in variability within each population can be compensated for by maintenance of diversity among populations [28-31]. Each environment selects for different alleles, allowing maintenance of all alleles in the metapopulation, even if some are lost at the individual population level. The principle of diversifying selection is used in genetic conservation studies.

\section{The Consequences of Phenotypic Plasticity on Genetic Diversity}

Phenotypic plasticity occurs at the individual level by changing the phenotype depending on the environment [10, 32-47]. For a given trait, this change with environment can be continuous or discrete [37]. In case of continuous phenotypic changes with an environment, the relationship between environmental variables and traits is called the reaction norms. These reaction norms are often complex and nonlinear. A plant or animal with a plastic trait receives a signal from the environment that will determine the trait value $[36,48-50]$. The phenotypic changes are implemented during the lifespan of an individual; that is, the effects are seen within one generation; this is not the case for selection.

Phenotypic changes of individuals within a population will lead to a modification of the distribution of the phenotypic values of this population within one generation (Figure 2). This modification is reversible without any genetic changes but in some cases this modification is conserved over several generations. Therefore, if the shift of phenotypic values is beneficial to fitness (i.e., adaptive) this adaptation 


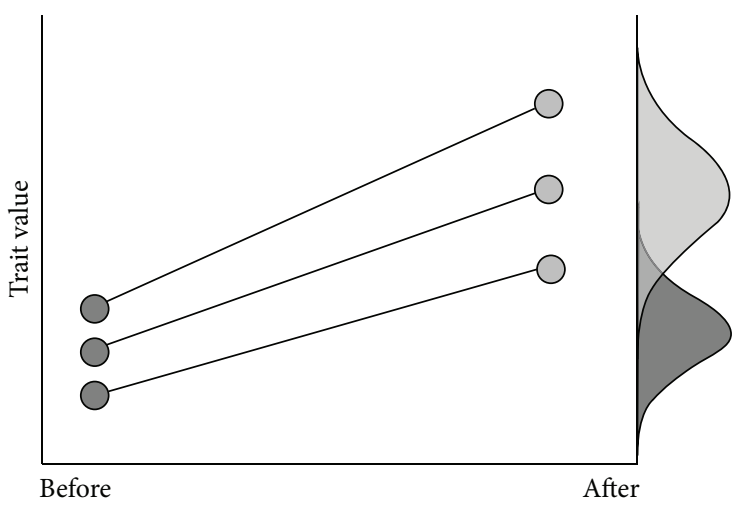

(a)

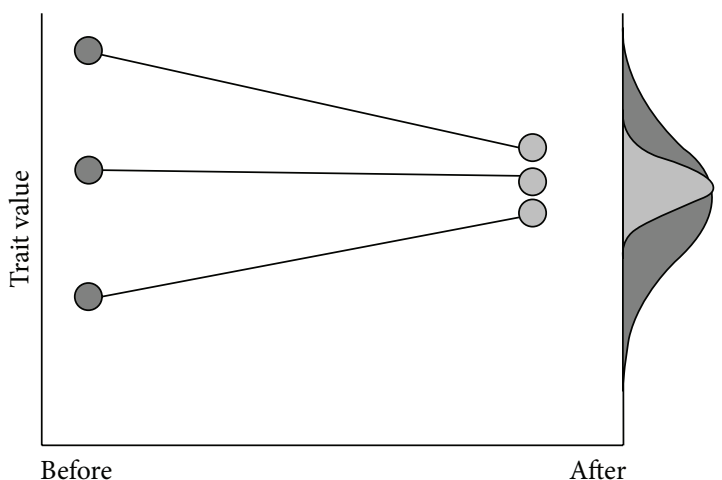

(b)

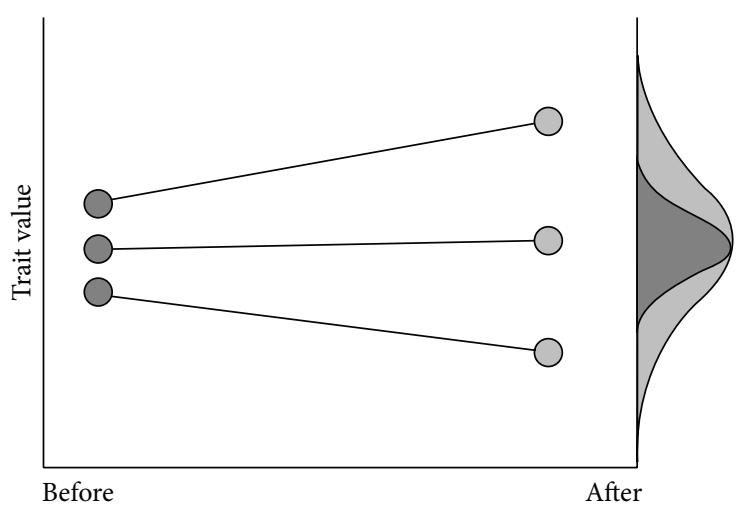

(c)

FIGURE 2: Scenarios for change in the mean and/or variance of a trait in a population between constitutive phenotypes expressed prior to an interaction (dark shading) and the induced phenotype following an interaction (light shading). (a) An increase in mean and variance of a trait. (b) A decrease in variance, mean unchanged. (c) An increase in variance, mean unchanged from [17].

can be obtained without a decrease in genetic diversity (Figure 1). In this case the phenotypic plasticity is adaptive and moderates the effects of selection. Some genotypes, not genetically adapted to an environment, become adapted through phenotypic plasticity and thus increase their fitness. Here it is necessary to introduce the notion of an optimal phenotype for a given environment. This is the phenotype which leads to the best fitness within the population. This optimal phenotype can be obtained with or without phenotypic plasticity. In a particular environment, if a genotype shows a phenotype close to the optimal phenotype, and if this phenotype is close to the average value of this genotype within a range of environments, it will have been obtained without phenotypic plasticity. In contrast, if another genotype shows a phenotype close to the optimal phenotype, and if this phenotype is far from being the average value of this genotype within a range of environments, then this phenotype will have been obtained through phenotypic plasticity. Within a population, the ability of phenotypic plasticity to moderate selection will depend on (i) the diversity of the traits involved in the optimal phenotype, (ii) the level of plasticity of the traits involved in the optimal phenotype, and (iii) the cost of the plasticity required to reach the optimal phenotype. This cost can be defined as the difference in fitness between the genotypes showing the optimal phenotype without plasticity and that of the genotypes displaying the optimal phenotype through plasticity. If the cost of plasticity is low enough, so that plastic genotypes have fitness as good as the best nonplastic genotypes, then the plastic genotypes will be selected for in the next generation. In such cases, with different levels of plasticity among genotypes (Figure 3), selection for plasticity will occur and will lead to a decrease in genetic diversity on the loci involved in plasticity variability. These can be different from the loci involved in the variability of the trait. Scheiner and Lyman [44] have shown in Drosophila that the plasticity of a trait can respond to selection and that this response is at least partially independent of the response of the trait mean. However, this independence remains controversial [51-53]. Within a population subject to different environments, adaptation over several generations comes from a balance between phenotypic plasticity and selection for both the value of adaptive traits and their plasticity. It is extremely rare to find studies that separate and quantify the roles: (1) of selection for the traits involved in the optimal phenotype, (2) of plasticity for these traits, and (3) of selection for plasticity of these traits. Nevertheless, each of these mechanisms has a different effect on the genetic diversity of the population. 


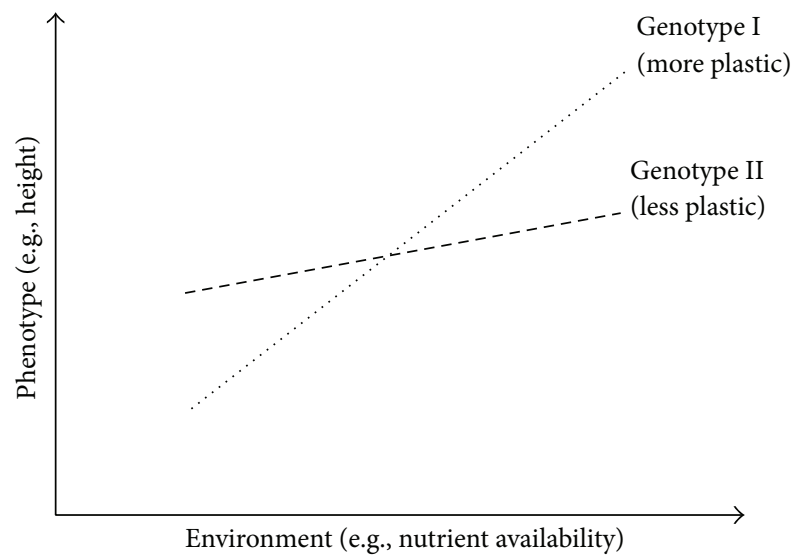

FIGURE 3: The lines represent the reaction norms (i.e. the genotypespecific environment-phenotype functions) of each genotype. Both lines have a slope in the environment-phenotype space, which means that both genotypes are plastic. The population shows GxE in the sense that there is genetic variation for the slope of the reaction norm, which would be detected by a standard analysis of variance from [18].

\section{Cost of plasticity}

Phenotypic plasticity can act on fitness [54] which can become either closer to or further from the optimal phenotype [43]. Depending on the effect of plasticity on individual fitness, plasticity can be characterised by a number of terms. Phenotypic plasticity is termed as "maladaptive" when the effect on fitness is negative, "neutral" if it has no effect on fitness, and "adaptive" when it has a positive effect on fitness [35]. There are various examples of adaptive plastic responses. Thus, a plastic response has been shown in the case of a ciliate species following the introduction of a predator. The ciliate developed lateral wings which increased its survival by inhibiting ingestion by the predator. In turn, the predator responded plastically by increasing its gape size to accommodate the ciliate's defence without cancelling it (mutual plasticity) [3]. Some examples of adaptive plastic responses in plants are the lengthening of internodes in the shade to better access light [55], various herbivoreresistance mechanisms [56], or adjustments to the number of simultaneously mature flowers depending on the number of pollinators present [57]. In some cases, adaptive plasticity can become maladaptive if the environment becomes too stressful [43]. For example, when competition for light is very strong, some plants can increase their internode length but if the neighbouring plant species are too tall, the plastic capacity of increasing internode length for the smaller genotypes becomes maladaptive [58-60].

Even adaptive phenotypic plasticity could come at a cost $[61,62]$. Lind and Johansson [63] suggest the cost increases with the level of plasticity in the population. This cost depends on genotype, population, and environment and can have quite a large number of origins [19]. These may include (i) maintenance costs (sensory structure, metabolic regulation mechanism of plasticity) [64], (ii) production costs (a plasticity-developed structure could be more costly than a genetically determined one) [65], (iii) acquisition of information costs (the process may be risky or it requires energy or reduces feeding or breeding efficiency), (iv) cost of imprecise development (nonspecific plastic change), (v) genetic costs (genetic linkage with deleterious alleles at other loci, pleiotropy, and epistasis) [66], and ecological costs (interaction between species; e.g., the plastic response to herbivory reduces attractiveness to pollinators) [67].

The costs of plasticity are difficult to quantify [68-70], but they can be estimated by comparing the fitness of plastic genotypes to that of fixed genotypes, for the same phenotypic value in each environment using the multiple regression: $W_{j}=$ constant $+\left(B_{1} \times Z_{j}\right)+\left(B_{2} \times P_{j}\right)$, where $W_{j}$ is the fitness of family $(j)$ in one environment, $B_{1}$ is the selection coefficient on phenotypes, $B_{2}$ is the selection coefficient on plasticity, $Z_{j}$ is the phenotype exhibited by family $j$ in one environment, and $P_{j}$ is the plasticity exhibited by family $j$ in different environments [71-73]. A significantly negative regression coefficient of the plasticity term indicates a genetic cost. This cost could be different in each environment. DeWitt et al. [19] adopt this method and analyse it to quantify the relationship between the phenotype and the fitness in each environment by regression analysis or by cubic splines [74]. This adaptation has the advantage of allowing nonlinear relationships between phenotype and fitness. This mathematical relationship allows calculation of a theoretical fitness that can be compared with the observed one. To visualise the trend, the residues of the fitness equation are shown graphically, based on the genotype degree of plasticity. There is a cost when the most plastic genotypes tend to have a lower fitness than expected and also when the fixed genotypes tend to have a higher fitness than expected (Figure 4). This method is interesting but a bias occurs when the phenotypic values are correlated with phenotypic plasticity [75]. This correlation must therefore be confirmed before calculating the cost of plasticity using this method. In several studies, the most extreme phenotypes have been associated with high plasticity $[56,73]$, which requires a reinterpretation of the results. If costs of plasticity are very high, they may have a considerable impact on the evolution of plasticity $[14,71,76,77]$. Although as indicated by the analysis of Van Buskirk and Steiner [78] in both animals and plants, costs of plasticity are often low and can influence phenotype evolution under stressful conditions only. The ubiquity of plasticity suggests the associated benefits outweigh the costs under a wide range of conditions [10].

\section{Selection, Plasticity, and Diversity: A Balance between Gain of Fitness and Cost}

If the plastic response is close to the optimal phenotype, the cost of achieving the phenotype by plasticity will determine the impact of selection on the plastic individual and therefore on allelic diversity of loci involved in trait value. If the cost of plasticity is significant, then achieving the optimal phenotype via fixed development is better than doing so via phenotypic plasticity. Individuals having a mutation that achieves the optimal phenotype directly will be selected in a stable environment. There is a kind of genetic evolution that results in reducing plasticity and in reducing the diversity of 


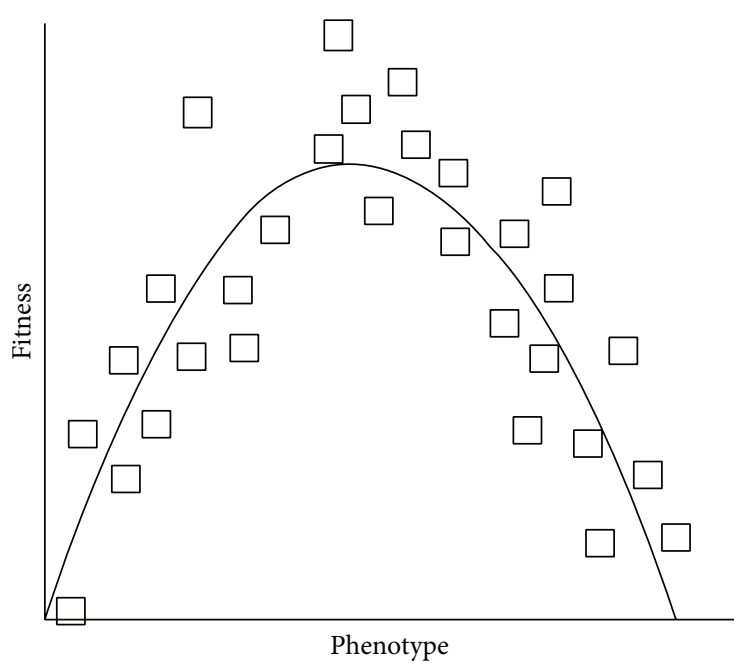

(a)

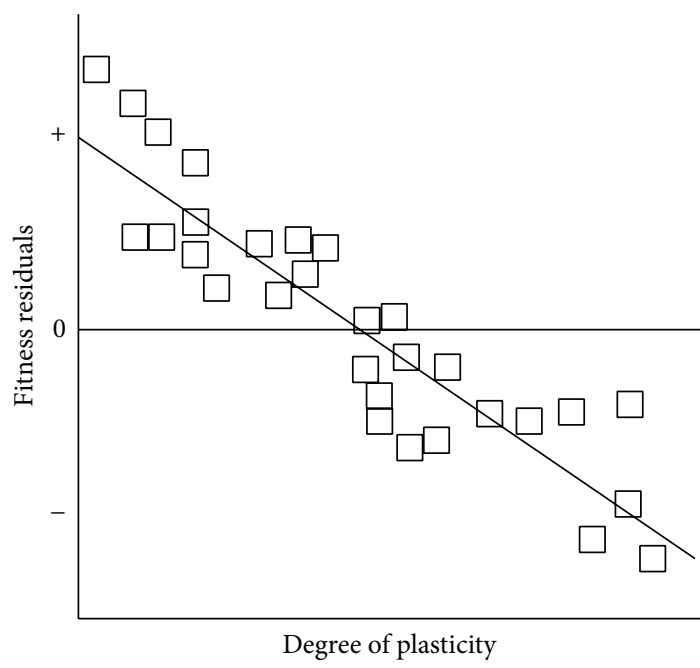

(b)

Figure 4: (a) Relationship between phenotypic values and fitness in one environment for population genotypes. (b) Relationship between degree of plasticity and fitness residuals. There is a cost of plasticity when the slope of the regression is negative from [19].

a trait. Due to the cost of plasticity, the evolution of plasticity is slower than evolution due to maladaptive plasticity (see above) for which the selection intensity is generally stronger.

If the plastic response is still some way off from being the optimal phenotype but is still favourable, a genetic evolution by directional selection can complete it and bring it closer to the optimal phenotype. In this case, to obtain the phenotype, there is both a plastic response and a change related to selection [54].

However, depending on the conditions encountered by populations and their initial states, several hypotheses can be formulated for adaptive plasticity. Plasticity can, for example, moderate the effects of natural selection by allowing individuals to adapt phenotypically to new conditions quickly. Chevin et al. [79] have developed a model to predict population persistence in a changing environment. It can estimate how plasticity might interfere with the capacities of populations to evolve in response to environmental change.

By reducing the response to selection, phenotypic plasticity may promote the maintenance of genetic diversity within a population. If individuals have plastic traits, they can adjust their phenotypes to the environment. Different genotypes with several different phenotypes in one environment may have the same phenotype in another environment and therefore some genetic variability is maintained. Genetic variations that are few or are not expressed in an environment due to plasticity [80] become neutral if there is no cost of plasticity and thus they avoid a decrease in fitness. These predictions emerge from theoretical models $[35,81,82]$ but there is little experimental data to verify them $[8,9]$. In Quercus coccifera, a tree species with several ecotypes, Baquedano et al. [83] show that phenotype plasticity hides differences between genotypes which maintain a genetic variability that is inaccessible to selection.
However, we can find populations having low genetic diversity that are maintained with the help of plasticity. Plasticity can allow morphological adaptation without the need for genetic variability. The study by Geng et al. [46] compares two species in which morphological variance is small. The morphological variance in one of the species is due mainly to genotypic variations, while in the other species, which has low genetic diversity, the morphological variance is due to plasticity.

As plasticity influences genetic changes by affecting the intensity of selection, we can expect an overall change in genetic diversity through reorganisation of the evolutionary dynamics and thus of the evolution rate [84]. Intensity of selection varies with plasticity, thus upsetting the expected evolution in the absence of plasticity $[17,85,86]$. In a population of lizards (Urosaurus ornatus), a directional selection has been shown to favour the fastest-moving males. However, a seasonal plasticity increases the fitness of the slowest individuals during the breeding season. This seasonal plasticity homogenises the speed of the male population at breeding time and reduces the effect of selection for high speed [87].

To assess the impact of selection and plasticity on the evolution of genetic diversity, models allow us to assess the rate of the genetic changes caused by plasticity. Hinton and Nowlan [88] developed a model that shows how learning increases the rate of evolution. This model was modified by Behera and Nanjundiah [89] to obtain greater realism. They used a haploid population of fixed size, different levels of plasticity, and a cost associated with the plasticity. Their results indicate that plasticity slows down the pace of evolution but enhances the level of adaptation. However, other models of the same type, which explored more parameters, obtained mixed results [90-92]. To estimate the evolution rates, Ancel [93] used a quantitative model and a reaction norm 


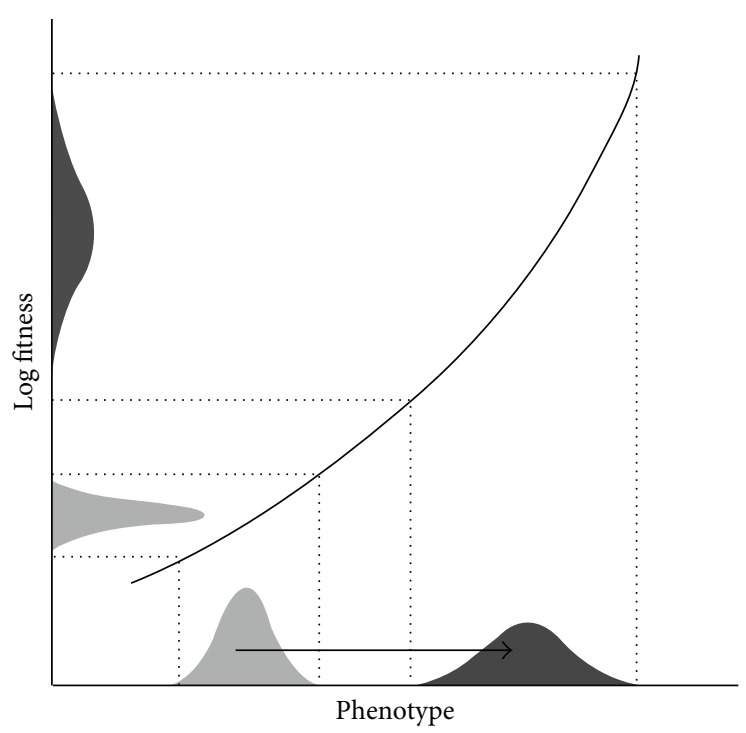

(a)

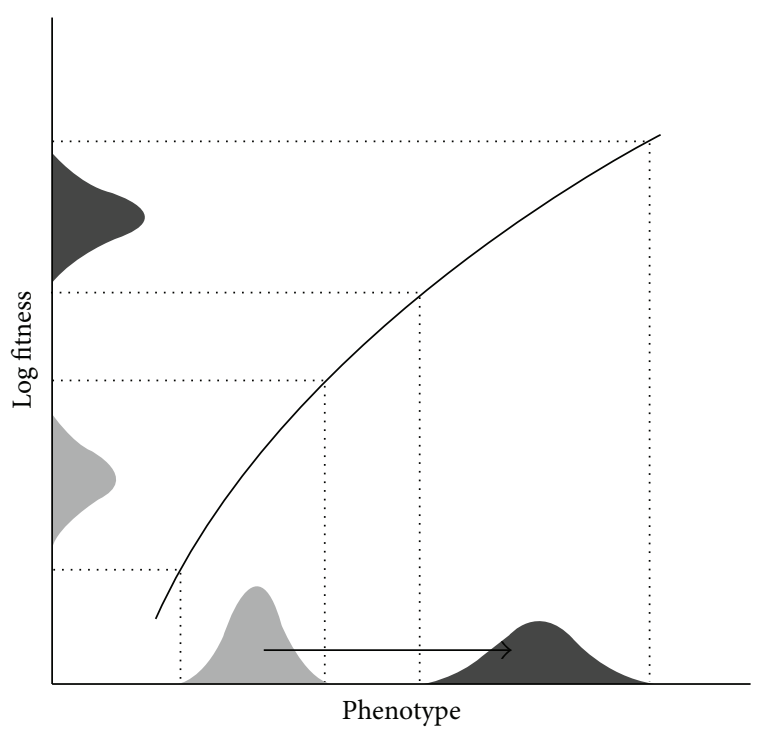

(b)

FIGURE 5: Schematic representation of the directional plasticity effects on the variation in the genotypic values $x$. The solid line shows the $\log$ of the fitness function, and the shaded areas symbolize the distributions of phenotypes and fitness without plasticity (light shading) and with plasticity (dark shading). Directional plasticity (symbolized by an arrow) shifts the distribution of the phenotypes (mean and variance). The curvature of the log fitness function (convex in (a); concave in (b)) determines how the shift in the mean of the distribution changes the variation in $\log$ fitness from [20].

model (modelling of plasticity by reaction norm instead of by genotype, phenotype points). Ancel's model is highly dependent on initial population conditions and on fitness. However, in most cases, the simulations obtained show that plasticity slows the evolution rate from the appearance, up to the stabilisation of the population at an optimal phenotype. When plasticity is not particularly costly, it protects suboptimal phenotypes from elimination by natural selection. In contrast, when plasticity is more costly, it fails to provide significant advantage. Furthermore, the phenotype ranges for which plasticity speeds up evolution are frequently those for which a plastic individual is less fit than its nonplastic counterpart. Paenke et al. [20] have sought conditions under which evolution is accelerated or delayed by plasticity under directional selection. According to these authors, the fitness gain gradient measures the effect of a marginal change in the degree of plasticity on the slope of the relationship between the genotypic value of the focal trait and $\log$ fitness. If the gain gradient has the same sign as the direction of selection, an increase in plasticity will magnify the response to selection. Conversely, if the two signs are opposite, greater plasticity will lead to a slower response. If plasticity acts similarly on genotypes, independently of their fitness, evolution is (i) accelerated if the logarithm of the fitness function (defined by the relationship between phenotype and fitness) follows a convex curve and (ii) slowed if this curve is concave (Figure 5). However, De Jong [94] considers the possibilities of evolution in the presence of plasticity and explains that there is no answer to the question of whether phenotypic plasticity promotes evolution. Everything depends on the case being studied and on the parameters associated with it.

\section{Conclusion}

The study of phenotypic plasticity has developed rapidly in recent decades, so we now have a clearer view of what it is and how it evolves. But for the interaction of selection and plasticity, more experimental work is required to better understand how selection acts on the reaction norm of phenotype against environments and also to better define the costs of plasticity. It should be noted that most studies focus on two environments. There is a scarcity of data on environment ranges, such as those commonly experienced by natural populations and on the changes in the selection pressures generated. In addition, measuring plasticity is difficult because the global plasticity scale of the individual is relative to the plasticity of the different traits of the individual [95]. Efforts should be made to estimate the global effect of plasticity on fitness of several traits. These studies would be improved with the development of a better understanding of the mechanisms involved, especially with regard to the genetic basis that explain plasticity. Despite numerous theoretical studies, there remains a lack of experimental data to support the results of these studies. Indeed, it is difficult to obtain experimental data in natural ecosystems where it is almost impossible to follow generations, to have replicated genotypes, and to control the environment. Ex situ experimentation could circumvent these limits by using (i) species which can be cloned or selfed and thus are easy to raise/grow and phenotype such as plant species, (ii) populations showing genetic diversity of adaptive traits and their plasticity, (iii) species with short life cycles, and (iv) semicontrolled environments. In the context of climate change, it would be interesting to conduct studies on 
crops showing these characteristics to define ways which can be used to maintain production under increasingly hazardous climates events.

\section{Competing Interests}

The authors declare that they have no competing interests.

\section{Acknowledgments}

The authors thank INRA and the region Poitou-Charentes for funding.

\section{References}

[1] R. Przybylo, B. C. Sheldon, and J. Merilä, "Climatic effects on breeding and morphology: evidence for phenotypic plasticity," Journal of Animal Ecology, vol. 69, no. 3, pp. 395-403, 2000.

[2] F. Valladares, D. Sanchez-Gomez, and M. A. Zavala, "Quantitative estimation of phenotypic plasticity: bridging the gap between the evolutionary concept and its ecological applications," Journal of Ecology, vol. 94, no. 6, pp. 1103-1116, 2006.

[3] M. Kopp and R. Tollrian, "Reciprocal phenotypic plasticity in a predator-prey system: inducible offences against inducible defences?" Ecology Letters, vol. 6, no. 8, pp. 742-748, 2003.

[4] T. E. Reed, D. E. Schindler, and R. S. Waples, "Interacting effects of phenotypic plasticity and evolution on population persistence in a changing climate," Conservation Biology, vol. 25, no. 1, pp. 56-63, 2011.

[5] B. W. Robinson and D. S. Wilson, "Genetic variation and phenotypic plasticity in a trophically polymorphic population of pumpkinseed sunfish (Lepomis gibbosus)," Evolutionary Ecology, vol. 10, no. 6, pp. 631-652, 1996.

[6] D. H. Nussey, E. Postma, P. Gienapp, and M. E. Visser, "Evolution: selection on heritable phenotypic plasticity in a wild bird population," Science, vol. 310, no. 5746, pp. 304-306, 2005.

[7] F. Aubret, X. Bonnet, and R. Shine, "The role of adaptive plasticity in a major evolutionary transition: early aquatic experience affects locomotor performance of terrestrial snakes," Functional Ecology, vol. 21, no. 6, pp. 1154-1161, 2007.

[8] S. E. Sultan and F. A. Bazzaz, "Phenotypic plasticity in Polygonum persicaria. II. Norms of reaction to soil moisture and the maintenance of genetic diversity," Evolution, vol. 47, no. 4, pp. 1032-1049, 1993.

[9] A. H. Cheetham, J. B. Jackson, and L. A. Hayek, "Quantitative genetics of bryozoan phenotypic evolution. III. Phenotypic plasticity and the maintenance of genetic variation," Evolution, vol. 49, no. 2, pp. 290-296, 1995.

[10] A. A. Agrawal, "Ecology-phenotypic plasticity in the interactions and evolution of species," Science, vol. 294, no. 5541, pp. 321-326, 2001.

[11] R. Bürger and M. Lynch, "Evolution and extinction in a changing environment: a quantitative-genetic analysis," Evolution, vol. 49, no. 1, pp. 151-163, 1995.

[12] S. N. Aitken, S. Yeaman, J. A. Holliday, T. Wang, and S. CurtisMcLane, "Adaptation, migration or extirpation: climate change outcomes for tree populations," Evolutionary Applications, vol. 1, no. 1, pp. 95-111, 2008.

[13] A. A. Hoffmann and C. M. Sgró, "Climate change and evolutionary adaptation,” Nature, vol. 470, no. 7335, pp. 479-485, 2011.
[14] S. E. Sultan and H. G. Spencer, "Metapopulation structure favors plasticity over local adaptation," The American Naturalist, vol. 160, no. 2, pp. 271-283, 2002.

[15] L. C. Latta IV, J. W. Bakelar, R. A. Knapp, and M. E. Pfrender, "Rapid evolution in response to introduced predators II: the contribution of adaptive plasticity," BMC Evolutionary Biology, vol. 7, article 21, 2007.

[16] G. Röder, M. Rahier, and R. E. Naisbit, "Counter-intuitive developmental plasticity induced by host quality," Proceedings of the Royal Society of London B: Biological Sciences, vol. 275, no. 1637, pp. 879-885, 2008.

[17] J. A. Fordyce, "The evolutionary consequences of ecological interactions mediated through phenotypic plasticity," Journal of Experimental Biology, vol. 209, no. 12, pp. 2377-2383, 2006.

[18] M. Pigliucci, "Evolution of phenotypic plasticity: where are we going now?" Trends in Ecology \& Evolution, vol. 20, no. 9, pp. 481-486, 2005.

[19] T. J. DeWitt, A. Sih, and D. S. Wilson, "Costs and limits of phenotypic plasticity," Trends in Ecology \& Evolution, vol. 13, no. 2, pp. 77-81, 1998.

[20] I. Paenke, B. Sendhoff, and T. J. Kawecki, "Influence of plasticity and learning on evolution under directional selection," The American naturalist, vol. 170, no. 2, pp. 47-58, 2007.

[21] C.-L. Meyer, A. A. Kostecka, P. Saumitou-Laprade et al., "Variability of zinc tolerance among and within populations of the pseudometallophyte species Arabidopsis halleri and possible role of directional selection," New Phytologist, vol. 185, no. 1, pp. 130-142, 2010.

[22] E. D. Brodie III, "Correlational selection for color pattern and antipredator behavior in the garter snake Thamnophis ordinoides," Evolution, vol. 46, no. 5, pp. 1284-1298, 1992.

[23] E. D. Brodie III and N. H. Russell, "The consistency of individual differences in behaviour: temperature effects on antipredator behaviour in garter snakes," Animal Behaviour, vol. 57, no. 2, pp. 445-451, 1999.

[24] M. E. N. Majerus, "Industrial melanism in the peppered moth, biston betularia: an excellent teaching example of darwinian evolution in action," Evolution: Education and Outreach, vol. 2, no. 1, pp. 63-74, 2009.

[25] H. E. Hoekstra, J. M. Hoekstra, D. Berrigan et al., "Strength and tempo of directional selection in the wild," Proceedings of the National Academy of Sciences of the United States of America, vol. 98, no. 16, pp. 9157-9160, 2001.

[26] X.-S. Zhang and W. G. Hill, "Competition can maintain genetic but not environmental variance in the presence of stabilizing selection," Evolution, vol. 61, no. 7, pp. 1532-1545, 2007.

[27] C. Rueffler, T. J. M. Van Dooren, O. Leimar, and P. A. Abrams, "Disruptive selection and then what?" Trends in Ecology and Evolution, vol. 21, no. 5, pp. 238-245, 2006.

[28] M. Nei, "Analysis of gene diversity in subdivided populations," Proceedings of the National Academy of Sciences of the United States of America, vol. 70, no. 12, pp. 3321-3323, 1973.

[29] K. A. Jolley, D. J. Wilson, P. Kriz, G. McVean, and M. C. J. Maiden, "The influence of mutation, recombination, population history, and selection on patterns of genetic diversity in Neisseria meningitidis," Molecular Biology and Evolution, vol. 22, no. 3, pp. 562-569, 2005.

[30] J. Van Heerwaarden, F. A. van Eeuwijk, and J. Ross-Ibarra, "Genetic diversity in a crop metapopulation," Heredity, vol. 104, no. 1, pp. 28-39, 2010. 
[31] A. M. Kotowska, J. F. Cahill Jr., and B. A. Keddie, "Plant genetic diversity yields increased plant productivity and herbivore performance," Journal of Ecology, vol. 98, no. 1, pp. 237-245, 2010.

[32] S. Via, "Adaptive phenotypic plasticity: target or by-product of selection in a variable environment?" The American Naturalist, vol. 142, no. 2, pp. 352-365, 1993.

[33] A. D. Bradshaw, "Evolutionary significance of phenotypic plasticity in plants," Advances in Genetics, vol. 13, pp. 115-155, 1965.

[34] C. Schlichting, "The evolution of phenotypic plasticity in plants," Annual Review of Ecology and Systematics, vol. 17, no. 1, pp. 667-693, 1986.

[35] S. M. Scheiner, "Genetics and evolution of phenotypic plasticity," Annual Review of Ecology and Systematics, vol. 24, pp. 3568, 1993.

[36] S. E. Sultan, "Phenotypic plasticity for plant development, function and life history," Trends in Plant Science, vol. 5, no. 12, pp. 537-542, 2000.

[37] V. Debat and P. David, "Mapping phenotypes: canalization, plasticity and developmental stability," Trends in Ecology and Evolution, vol. 16, no. 10, pp. 555-561, 2001.

[38] M. Pigliucci and A. Kolodynska, "Phenotypic plasticity to light intensity in Arabidopsis thaliana: invariance of reaction norms and phenotypic integration," Evolutionary Ecology, vol. 16, no. 1, pp. 27-47, 2002.

[39] B. G. Miner, S. E. Sultan, S. G. Morgan, D. K. Padilla, and R. A. Relyea, "Ecological consequences of phenotypic plasticity," Trends in Ecology and Evolution, vol. 20, no. 12, pp. 685-692, 2005.

[40] J. G. Swallow, J. S. Rhodes, and T. Garland Jr., "Phenotypic and evolutionary plasticity of organ masses in response to voluntary exercise in house mice," Integrative and Comparative Biology, vol. 45, no. 3, pp. 426-437, 2005.

[41] G. Gorur, C. Lomonaco, and A. Mackenzie, "Phenotypic plasticity in host-plant specialisation in Aphis fabae," Ecological Entomology, vol. 30, no. 6, pp. 657-664, 2005.

[42] M. J. West-Eberhard, "Phenotypic accommodation: adaptive innovation due to developmental plasticity," Journal of Experimental Zoology Part B: Molecular and Developmental Evolution, vol. 304, no. 6, pp. 610-618, 2005.

[43] C. K. Ghalambor, J. K. McKay, S. P. Carroll, and D. N. Reznick, "Adaptive versus non-adaptive phenotypic plasticity and the potential for contemporary adaptation in new environments," Functional Ecology, vol. 21, no. 3, pp. 394-407, 2007.

[44] S. Scheiner and R. Lyman, "The genetics of phenotypic plasticity. II. Response to selection," Journal of Evolutionary Biology, vol. 4, pp. 23-50, 1991.

[45] L. J. Chapman, F. Galis, and J. Shinn, "Phenotypic plasticity and the possible role of genetic assimilation: hypoxia-induced tradeoffs in the morphological traits of an African cichlid," Ecology Letters, vol. 3, no. 5, pp. 387-393, 2000.

[46] Y.-P. Geng, X.-Y. Pan, C.-Y. Xu, W.-J. Zhang, B. Li, and J.-K. Chen, "Phenotypic plasticity of invasive Alternanthera philoxeroides in relation to different water availability, compared to its native congener," Acta Oecologica, vol. 30, no. 3, pp. 380-385, 2006.

[47] S. I. Peluc, T. S. Sillett, J. T. Rotenberry, and C. K. Ghalambor, "Adaptive phenotypic plasticity in an island songbird exposed to a novel predation risk," Behavioral Ecology, vol. 19, no. 4, pp. 830-835, 2008.
[48] R. M. Borges, "Do plants and animals differ in phenotypic plasticity?” Journal of Biosciences, vol. 30, no. 1, pp. 41-50, 2005.

[49] P. Beldade, A. R. A. Mateus, and R. A. Keller, "Evolution and molecular mechanisms of adaptive developmental plasticity," Molecular Ecology, vol. 20, no. 7, pp. 1347-1363, 2011.

[50] A. B. Nicotra and A. Davidson, "Adaptive phenotypic plasticity and plant water use," Functional Plant Biology, vol. 37, no. 2, pp. 117-127, 2010.

[51] S. Via, R. Gomulkiewicz, G. De Jong, S. M. Scheiner, C. D. Schlichting, and P. H. van Tienderen, "Adaptive phenotypic plasticity: consensus and controversy," Trends in Ecology \& Evolution, vol. 10, no. 5, pp. 212-217, 1995.

[52] G. de Jong, "Phenotypic plasticity as a product of selection in a variable environment," The American Naturalist, vol. 145, no. 4, pp. 493-512, 1995.

[53] G. de Jong and P. Bijma, "Selection and phenotypic plasticity in evolutionary biology and animal breeding," Livestock Production Science, vol. 78, no. 3, pp. 195-214, 2002.

[54] T. D. Price, A. Qvarnström, and D. E. Irwin, "The role of phenotypic plasticity in driving genetic evolution," Proceedings of the Royal Society B: Biological Sciences, vol. 270, no. 1523, pp. 1433-1440, 2003.

[55] J. Schmitt, S. A. Dudley, and M. Pigliucci, "Manipulative approaches to testing adaptive plasticity: phytochromemediated shade-avoidance responses in plants," American Naturalist, vol. 154, pp. S43-S54, 1999.

[56] A. A. Agrawal, J. K. Conner, M. T. J. Johnson, and R. Wallsgrove, "Ecological genetics of an induced plant defense against herbivores: additive genetic variance and costs of phenotypic plasticity," Evolution, vol. 56, no. 11, pp. 2206-2213, 2002.

[57] L. D. Harder and S. D. Johnson, "Adaptive plasticity of floral display size in animal-pollinated plants," Proceedings of the Royal Society of London B: Biological Sciences, vol. 272, no. 1581, pp. 2651-2657, 2005.

[58] K. Donohue, D. Messiqua, E. H. Pyle, M. Shane Hesche, and J. Schmitt, "Evidence of adaptive divergence in plasticity: densityand site-dependent selection on shade-avoidance responses in impatiens capensis," Evolution, vol. 54, no. 6, pp. 1956-1968, 2000.

[59] C. Weinig, "Plasticity versus canalization: population differences in the timing of shade-avoidance responses," Evolution, vol. 54, no. 2, pp. 441-451, 2000.

[60] T. Steinger, B. A. Roy, and M. L. Stanton, "Evolution in stressful environments II: adaptive value and costs of plasticity in response to low light in Sinapis arvensis," Journal of Evolutionary Biology, vol. 16, no. 2, pp. 313-323, 2003.

[61] J. Merilä, A. Laurila, and B. Lindgren, "Variation in the degree and costs of adaptive phenotypic plasticity among Rana temporaria populations," Journal of Evolutionary Biology, vol. 17, no. 5, pp. 1132-1140, 2004.

[62] M. Van Kleunen and M. Fischer, "Constraints on the evolution of adaptive phenotypic plasticity in plants," New Phytologist, vol. 166, no. 1, pp. 49-60, 2005.

[63] M. I. Lind and F. Johansson, "Costs and limits of phenotypic plasticity in Island populations of the common frog Rana Temporaria under divergent selection pressures," Evolution, vol. 63, no. 6, pp. 1508-1518, 2009.

[64] P. Edelaar, T. Piersma, and E. Postma, "Retained non-adaptive plasticity: gene flow or small inherent costs of plasticity?" Evolutionary Ecology Research, vol. 7, no. 3, pp. 489-495, 2005. 
[65] B. Ernande and U. Dieckmann, "The evolution of phenotypic plasticity in spatially structured environments: implications of intraspecific competition, plasticity costs and environmental characteristics," Journal of Evolutionary Biology, vol. 17, no. 3, pp. 613-628, 2004.

[66] J. M. Dechaine, J. A. Johnston, M. T. Brock, and C. Weinig, "Constraints on the evolution of adaptive plasticity: costs of plasticity to density are expressed in segregating progenies," New Phytologist, vol. 176, no. 4, pp. 874-882, 2007.

[67] F. Valladares, E. Gianoli, and J. M. Gómez, "Ecological limits to plant phenotypic plasticity," New Phytologist, vol. 176, no. 4, pp. 749-763, 2007.

[68] T. J. DeWitt, "Costs and limits of phenotypic plasticity: tests with predator-induced morphology and life history in a freshwater snail," Journal of Evolutionary Biology, vol. 11, no. 4, pp. 465-480, 1998.

[69] J. Loman and D. Claesson, "Plastic response to pond drying in tadpoles Rana temporaria: tests of cost models," Evolutionary Ecology Research, vol. 5, no. 2, pp. 179-194, 2003.

[70] S. Volis, "Plasticity, its cost, and phenotypic selection under water and nutrient stress in two annual grasses," Biological Journal of the Linnean Society, vol. 97, no. 3, pp. 581-593, 2009.

[71] P. H. Van Tienderen, "Evolution of generalists and specialists in spatially heterogeneous environments," Evolution, vol. 45, no. 6, pp. 1317-1331, 1991.

[72] S. M. Scheiner and D. Berrigan, "The genetics of phenotypic plasticity. VIII. The cost of plasticity in Daphnia pulex," Evolution, vol. 52, no. 2, pp. 368-378, 1998.

[73] R. A. Relyea, "Costs of phenotypic plasticity," The American Naturalist, vol. 159, no. 3, pp. 272-282, 2002.

[74] E. D. Brodie III, A. J. Moore, and F. J. Janzen, "Visualizing and quantifying natural selection," Trends in Ecology \& Evolution, vol. 10, no. 8, pp. 313-318, 1995.

[75] J. R. Auld, A. A. Agrawal, and R. A. Relyea, "Re-evaluating the costs and limits of adaptive phenotypic plasticity," Proceedings of the Royal Society of London B: Biological Sciences, vol. 277, no. 1681, pp. 503-511, 2010.

[76] M. Lynch and W. Gabriel, "Environmental tolerance," The American Naturalist, vol. 129, no. 2, pp. 283-303, 1987.

[77] N. A. Moran, "The evolutionary maintenance of alternative phenotypes," The American Naturalist, vol. 139, no. 5, pp. 971989, 1992.

[78] J. Van Buskirk and U. K. Steiner, "The fitness costs of developmental canalization and plasticity," Journal of Evolutionary Biology, vol. 22, no. 4, pp. 852-860, 2009.

[79] L.-M. Chevin, R. Lande, and G. M. Mace, "Adaptation, plasticity, and extinction in a changing environment: towards a predictive theory," PLoS Biology, vol. 8, no. 4, Article ID e1000357, 2010.

[80] C. D. Schlichting and H. Smith, "Phenotypic plasticity: linking molecular mechanisms with evolutionary outcomes," Evolutionary Ecology, vol. 16, no. 3, pp. 189-211, 2002.

[81] J. H. Gillespie and M. Turelli, "Genotype-environment interactions and the maintenance of polygenic variation," Genetics, vol. 121, no. 1, pp. 129-138, 1989.

[82] L. A. Zhivotovsky and S. Gavrilets, "Quantitative variability and multilocus polymorphism under epistatic selection," Theoretical Population Biology, vol. 42, no. 3, pp. 254-283, 1992.

[83] F. J. Baquedano, F. Valladares, and F. J. Castillo, "Phenotypic plasticity blurs ecotypic divergence in the response of Quercus coccifera and Pinus halepensis to water stress," European Journal of Forest Research, vol. 127, no. 6, pp. 495-506, 2008.
[84] R. A. Duckworth, "The role of behavior in evolution: a search for mechanism," Evolutionary Ecology, vol. 23, no. 4, pp. 513531, 2009.

[85] T. D. Price, "Phenotypic plasticity, sexual selection and the evolution of colour patterns," The Journal of Experimental Biology, vol. 209, no. 12, pp. 2368-2376, 2006.

[86] M. J. West-Eberhard, "Phenotypic plasticity and the origins of diversity," Annual Review of Ecology and Systematics, vol. 20, pp. 249-278, 1989.

[87] D. J. Irschick and J. J. Meyers, "An analysis of the relative roles of plasticity and natural selection in the morphology and performance of a lizard (Urosaurus ornatus)," Oecologia, vol. 153, no. 2, pp. 489-499, 2007.

[88] G. E. Hinton and S. J. Nowlan, "How learning can guide evolution," Complex Systems, vol. 1, pp. 495-502, 1987.

[89] N. Behera and V. Nanjundiah, "An investigation into the role of phenotypic plasticity in evolution," Journal of Theoretical Biology, vol. 172, no. 3, pp. 225-234, 1995.

[90] S. Nolfi and D. Floreano, "Learning and evolution," Autonomous Robots, vol. 7, no. 1, pp. 89-113, 1999.

[91] H. Dopazo, M. B. Gordon, R. Perazzo, and S. Risau-Gusman, "A model for the interaction of learning and evolution," Bulletin of Mathematical Biology, vol. 63, no. 1, pp. 117-134, 2001.

[92] E. Borenstein, I. Meilijson, and E. Ruppin, "The effect of phenotypic plasticity on evolution in multipeaked fitness landscapes," Journal of Evolutionary Biology, vol. 19, no. 5, pp. 1555-1570, 2006.

[93] L. W. Ancel, "Undermining the Baldwin expediting effect: does phenotypic plasticity accelerate evolution?" Theoretical Population Biology, vol. 58, no. 4, pp. 307-319, 2000.

[94] G. De Jong, "Evolution of phenotypic plasticity: patterns of plasticity and the emergence of ecotypes," New Phytologist, vol. 166, no. 1, pp. 101-118, 2005.

[95] H. De Kroon, H. Huber, J. F. Stuefer, and J. M. van Groenendael, "A modular concept of phenotypic plasticity in plants," New Phytologist, vol. 166, no. 1, pp. 73-82, 2005. 

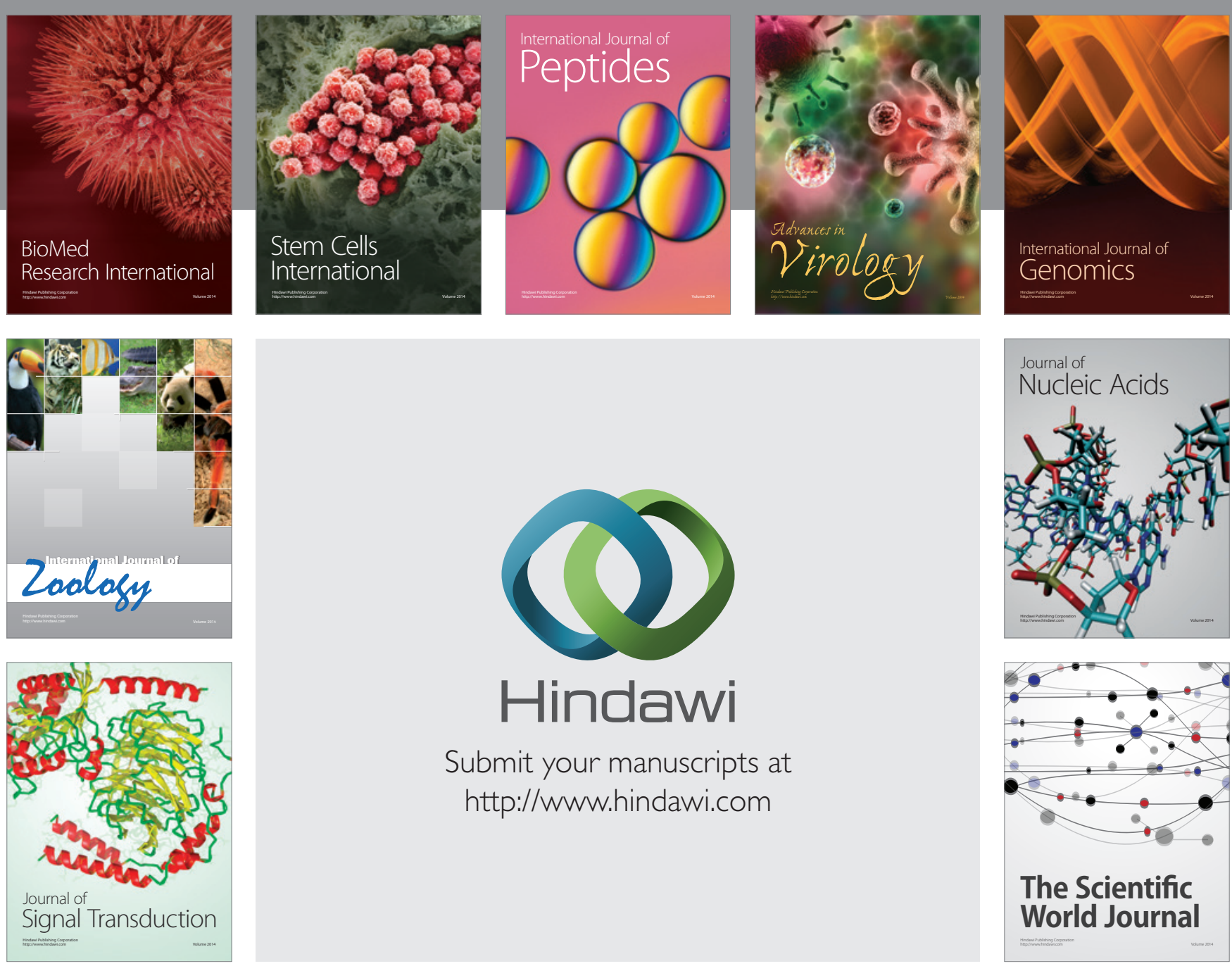

Submit your manuscripts at

http://www.hindawi.com
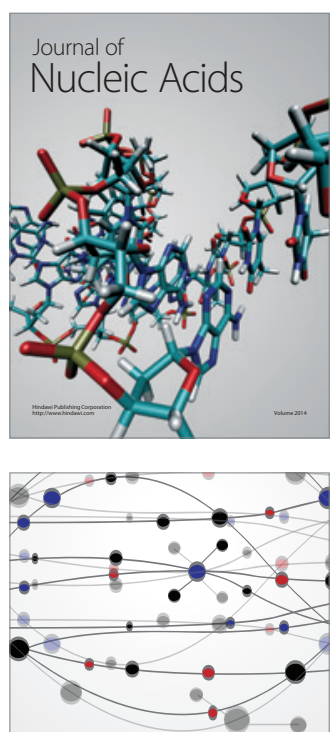

The Scientific World Journal
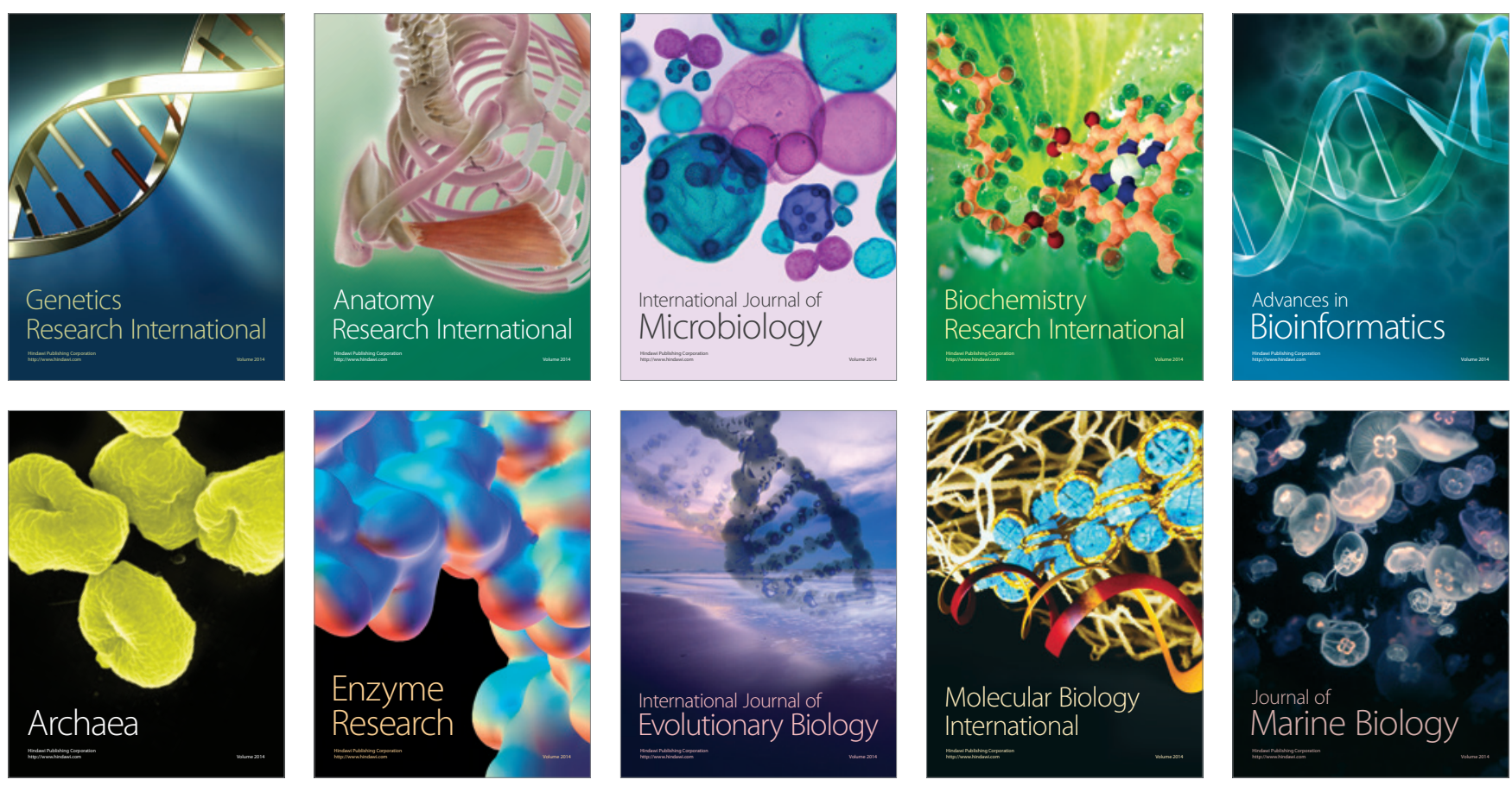